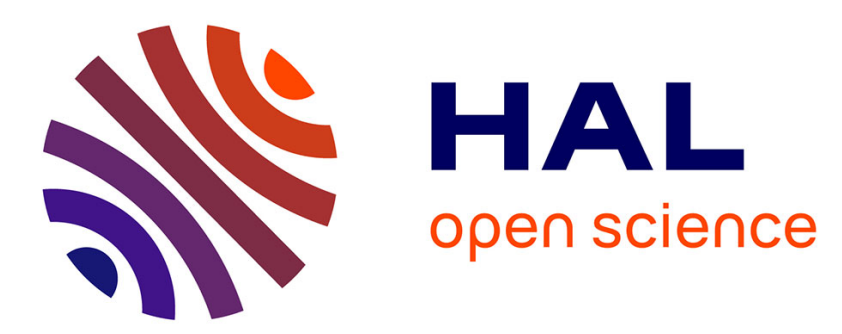

\title{
Comparison of turbulence closures for optimized active control
}

Régis Duvigneau, Jérémie Labroquère, Emmanuel Guilmineau

\section{To cite this version:}

Régis Duvigneau, Jérémie Labroquère, Emmanuel Guilmineau. Comparison of turbulence closures for optimized active control. Computers and Fluids, 2016, 124 (124), pp.67-77. 10.1016/j.compfluid.2015.10.011 . hal-01251823

HAL Id: hal-01251823

https://hal.inria.fr/hal-01251823

Submitted on 6 Jan 2016

HAL is a multi-disciplinary open access archive for the deposit and dissemination of scientific research documents, whether they are published or not. The documents may come from teaching and research institutions in France or abroad, or from public or private research centers.
L'archive ouverte pluridisciplinaire HAL, est destinée au dépôt et à la diffusion de documents scientifiques de niveau recherche, publiés ou non, émanant des établissements d'enseignement et de recherche français ou étrangers, des laboratoires publics ou privés. 


\title{
Comparison of turbulence closures for optimized active control
}

\author{
Régis Duvigneau $^{\mathrm{a}, *}$, Jérémie Labroquère ${ }^{\mathrm{a}}$, Emmanuel Guilmineau $^{\mathrm{b}}$ \\ ${ }^{a}$ INRIA Sophia-Antipolis Méditerranée, Opale Project-Team \\ 2004 route des lucioles, 06902 Sophia-Antipolis, France \\ ${ }^{b}$ Laboratoire de recherche en Hydrodynamique, Energétique et Environnement \\ Atmosphérique (LHEEA) CNRS UMR 6598 \\ Ecole Centrale de Nantes, rue de la Noë, 44321 Nantes, France
}

\begin{abstract}
Active flow control strategies, such as oscillatory blowing / suction, have proved their efficiency to modify flow characteristics for various purposes (e.g. skin friction reduction, separation delay, etc) in case of rather simple configurations. To extend this approach to industrial cases, the simulation of a large number of devices at real scale and the optimization of parameters are required. In this perspective, numerical simulations based on ReynoldsAveraged Navier-Stokes (RANS) equations seem to be the most appropriate framework, despite the well known limitations of turbulence closures in the context of unsteady separated flows. Thus, the objective of this work is to evaluate the ability of RANS models for the optimization of control devices and compare the results obtained using different turbulence closures. In this perspective, an incompressible RANS solver for unstructured grids is coupled with a surrogate-based global optimizer. The resulting tool is applied to derive an optimal actuation, based on an oscillatory blowing / suction device, for a set of turbulence closures including two-equation eddy-viscosity models and an explicit algebraic Reynolds stress model. As test-case, the reduction of the separation length for a backward facing step is targeted. Results are finally compared and analyzed, in terms of flow characteristics and optimal actuation parameters found.
\end{abstract}

Keywords: flow control, turbulence, optimization

\footnotetext{
${ }^{*}$ Corresponding author

Email address: Regis.Duvigneau@inria.fr (Régis Duvigneau)
} 


\section{Introduction}

Active flow control has been a growing research area for the last decades, since this approach demonstrated its ability to improve aerodynamic performance [13]. It is especially appealing in case of separated flows, for which natural instability phenomena can be efficiently exploited to manipulate flow characteristics. Among the possible active control strategies, periodic flow excitation has been particularly studied. Thus, several investigations have been carried out, experimentally as well as numerically, to apply this technology to various purposes, like separation delay [6, 12, 34], mixing enhancement [30], flow vectorization [36], etc.

Simulation has a critical role to play regarding the emergence of this approach for real-life applications, because the excitation parameters, such as actuator location, frequency, amplitude, should be set precisely to determine a flow control strategy, that is efficient and as inexpensive as possible. This task is usually problem dependent and setting a priori values for these parameters could be non-trivial, despite of the intuition of practitioners. To overcome this issue, the simulation of actuated flows can be used to determine optimal control parameters, or at least a range of efficient parameters. This task can be carried out by a systematic and parametric way [12], or by the use of an automated optimization procedure [3, 9, 10, 17, 38]. However, simulating actuated flows is also a difficult exercise. It has been shown, in particular during the CFDVAL Workshop [32], that the prediction of actuator-induced flows is still tedious, because of the difficulty to model the flow in the vicinity of the actuator and in the separated regions. Even if Large Eddy Simulation (LES) methods usually outclass Reynolds-Averaged Navier-Stokes (RANS) models for such problems, LES methods are still unable to predict actuated flows in a computational time that would be reasonable in an industrial context. In a recent study [14], four RANS and one LES models have been compared in the case of a separated flow including a synthetic jet actuator, for a set of thirteen frequencies. It has been found that RANS models are not able to predict the correct characteristics of the separation, but some of them are able to identify the best actuation frequency, among those tested, to reduce the separation length. The decrease of the recirculation length is under-estimated by RANS models, but the ranking of the actuation parameters has been correctly established. 
This encouraging result indicates that RANS models could possibly be used in a design phase to select control parameters. Therefore, we propose in the current work a similar study, in which a full optimization of actuation parameters is performed, for a set of RANS models, in a separated flow context. The objective is to assess the ability of RANS models to be used in an optimization framework for active flow control. We underline that, unfortunately, it is not possible presently to carry out an optimization campaign using LES for high Reynolds numbers, for reference purpose. It is even not possible to confirm that a given actuation is optimal on the basis of such models, because it would require several neighboring results. Finally, optimality conditions are difficult to establish experimentally. This explains why our optimization study is restricted to RANS models, after comparison with experimental results for the baseline flow.

The paper is organized in five main parts: in a first section, the numerical models employed for the flow and the actuation are described. In a second section, we present the surrogate-based global optimizer used to determine optimal control parameters. In a third section, the backward facing step test-case considered here, with an oscillatory actuation, is described. The baseline flow without actuation is presented in section four, including a grid refinement study and comparisons between experimental data and different RANS models. Finally, in the sections five and six, the results of the optimization procedure are analyzed and discussed.

\section{Numerical models}

\subsection{Flow model}

This work is restricted to incompressible flow studies, carried out using the ISIS-CFD solver, developed at LHEEA and available as a part of the FINE/Marine computing suite. It solves incompressible Unsteady ReynoldsAveraged Navier-Stokes (URANS) equations. The solver is based on a finitevolume method to build the spatial discretization, the conservation equations being solved by a face-based cell-centered approach.

Within this framework, the incompressible conservation laws under isothermal conditions are written as:

$$
\frac{\partial}{\partial t} \int_{V} \rho d V+\int_{S} \rho \vec{U} \cdot \vec{n} d S=0
$$




$$
\frac{\partial}{\partial t} \int_{V} \rho U_{i} d V+\int_{S} \rho U_{i} \vec{U} \cdot \vec{n} d S=\int_{S}\left(\tau_{i j} I_{j}-p I_{i}\right) \cdot \vec{n} d S,
$$

where $\mathrm{V}$ is the domain of interest or control volume, bounded by the closed surface $\mathrm{S}$ with a unit normal vector $\vec{n}$ directed outward. $\vec{U}$ and $p$ represent, respectively, the velocity and pressure fields. $\tau_{i j}$ are the components of the viscous stress tensor, whereas $I_{j}$ is a vector whose components are zero, except for the $j^{\text {th }}$ component which is equal to unity.

All flow variables are stored at geometric centers of the arbitrary shaped cells. Surface and volume integrals are evaluated according to second-order accurate approximations by using the values of integrand that prevail at the center of the face $f$, or cell $C$, and neighbor cells $C_{n b}$. The various fluxes appearing in the discretized equations (1) and (2) are built using the AVLSMART scheme [27] in the Normalized Variable Diagram (NVD) context [28]. More details can be found in [11] and more recently in [28]. A pressure equation is obtained in the spirit of the Rhie and Chow SIMPLE (Semi-Implicit Method for Pressure Linked Equations) algorithm [29]. Unsteady terms are solved using a second-order dual-time stepping approach [18].

In the case of turbulent flows, additional transport equations for modeled variables are discretized and solved using the same principles.

\subsection{Turbulence closures}

In this work, we study the ability of RANS models to predict meaningful information for the optimization of control devices in industrial context. Therefore, turbulence closure is restricted to classical linear eddy-viscosity or non-linear algebraic stress models. More specifically, we consider the twoequation k- $\epsilon$ model from Launder-Spalding [22], the SST (Shear-Stress Transport) $k-\omega$ model from Menter [25], and finally the algebraic stress model based on a $k-\omega$ closure from Gatski \& Speziale [15] with a near-wall formulation [5]. All the above mentioned models are implemented according to their standard version and we refer to the bibliography for additional details.

\subsection{Actuator model}

We consider in this study a zero-mass net oscillatory actuation. Our main objective is to determine the optimal actuated flow and its dependency with respect to turbulence closures, independently from the actuation process itself. Therefore, the detailed modeling of the actuator is not targeted here and we restrict the actuator model to an imposed velocity boundary condition:

$$
\vec{U}=U_{j} A(\mathbf{x}) \sin \left(2 \pi f_{j} t\right) \vec{d}_{j}
$$


with $\mathrm{A}(\mathbf{x})$ a unitary profile (squared sine function), $U_{j}$ the amplitude, $f_{j}$ the frequency and $\vec{d}_{j}$ a unit vector normal to the boundary. For turbulent variables, a zero-Neumann boundary condition is prescribed. Obviously, this actuation corresponds to a simplified model of synthetic jet, for which an oscillatory cavity generates the suction / blowing effect[16, 36]. As shown in several studies $[6,20,31,32,35,37]$, the accurate prediction of the flow characteristics at the exit slot is a difficult task, beyond the objectives of the present work.

\section{Optimization method}

To determine optimal actuation parameters, the flow solver is coupled to an optimization algorithm that seeks the minimization of a cost functional. In the context of unsteady turbulent flows, optimization is not an easy task. In particular, the unsteadiness makes the use of classical descent methods based on the adjoint system tedious. Moreover, the computational time makes the use of alternate "black-box" optimizers like genetic algorithms practically impossible. Therefore, we adopt here an optimization strategy based on an adaptive statistical learning approach. The method has already been described and validated for flow problems in [8], and used by other authors in $[1,23]$. Nevertheless, we describe below its main features, since some of them will impact the results regarding the optimization of control devices.

This method is based on the construction of Gaussian Process models [33] (also known as kriging models). Such models allow to predict a function value $f$ at a given point $x$, on the basis of a set of observed values $F_{N}=$ $\left\{f_{1}, f_{2}, \ldots, f_{N}\right\}$ corresponding to the points $X_{N}=\left\{x_{1}, x_{2}, \ldots, x_{N}\right\}$. Since the function is not known, a statistical framework is adopted and $F_{N}$ is assumed to be a realization of a multivariate Gaussian process, which has a joint Gaussian distribution:

$$
p\left(F_{N} \mid X_{N}\right)=\frac{\exp \left(-\frac{1}{2} F_{N}^{\top} C_{N}^{-1} F_{N}\right)}{\sqrt{(2 \pi)^{N} \operatorname{det}\left(C_{N}\right)}},
$$

for any inputs $X_{N}$. $C_{N}$ is the $N \times N$ covariance matrix, whose elements $C_{m n}$ describe the correlation between the function values $f_{m}$ and $f_{n}$ obtained at points $x_{m}$ and $x_{n}$. This is expressed in terms of a correlation function $k$, i.e., 
$C_{m n}=\operatorname{cov}\left(f_{m}, f_{n}\right)=k\left(x_{m}, x_{n} ; \Theta\right)$ with $\Theta$ a set of hyper-parameters used for model calibration.

Using conditional probabilities, it can be shown [33] that the function value $f_{N+1}$ at a new point $x_{N+1}$ has a joint probability density:

$$
p\left(f_{N+1} \mid\left(X_{N}, F_{N}\right), x_{N+1}\right) \propto \exp \left[-\frac{\left(f_{N+1}-\hat{f}_{N+1}\right)^{2}}{2 \sigma_{f_{N+1}}^{2}}\right],
$$

where

$$
\hat{f}_{N+1}=K_{N+1}^{\top} C_{N}^{-1} F_{N}, \quad \hat{\sigma}_{f_{N+1}}^{2}=\kappa-K_{N+1}^{\top} C_{N}^{-1} K_{N+1},
$$

with $\kappa=k\left(x_{N+1}, x_{N+1} ; \Theta\right)$ and $K_{N+1}=\left[k\left(x_{1}, x_{N+1} ; \Theta\right), \ldots, k\left(x_{N}, x_{N+1} ; \Theta\right)\right]^{T}$. Thus, the probability density for the function value at the new point $x_{N+1}$ is Gaussian with mean $\hat{f}_{N+1}$ and standard deviation $\hat{\sigma}_{f_{N+1}}$. Therefore, the most likely value at the new point $x_{N+1}$ is $\hat{f}_{N+1}$. This value will be considered as the prediction of the Gaussian process model. The variance $\hat{\sigma}_{f_{N+1}}^{2}$ can be interpreted as a measure of uncertainty in the value prediction.

The choice of the correlation function $k$ is critical for the model, as it contains all the prior hypotheses, including for example regularity, symmetry, periodicity, etc. More specifically, a classical anisotropic multidimensional correlation function is employed here:

$$
k\left(x, x^{\prime} ; \Theta\right)=\theta_{1} \prod_{i=1}^{d} e^{-\left(\frac{x_{i}-x_{i}^{\prime}}{r_{i}}\right)^{2}}+\theta_{2},
$$

with $d$ the number of design variables, $\Theta=\left\{\theta_{1}, \theta_{2}, r_{1}, r_{2}, \ldots, r_{d}\right\}$ a set of hyper-parameters, which is determined by maximizing the probability density function of the observed data, given by equation (4).

A first Design Of Experiments (DOE) phase is carried out to construct an initial model. Then, an iterative and adaptive strategy is used to enrich the model and locate the most interesting values of the cost functional [19]. In this perspective, an internal optimization problem is solved to determine a set of new points to be evaluated, by minimizing or maximizing a so-called merit function, which is based on both model value and variance. A comprehensive review of the different merit functions to locate the optimum of deterministic functions is provided in [19]. In the present study, the model enrichment is based on the lower bound criterion, which consists in minimizing the quantity $\hat{f}-\rho \hat{\sigma}_{f}$, the parameter $\rho=0,1,2$ being used to balance the 
search for the minimum and the exploration of uncertain regions. The new cost functional evaluations are then employed to update the model. This enrichment procedure is conducted until convergence. Implementation details can be found in $[4,8]$. Note that this optimization approach allows to filter a possible noise, arising in the evaluation process, by considering the simulation outputs as noisy observations $[21,26]$. In this perspective, the cost function value at $x_{i}$ is considered as an observation including a noise $f_{i}=f\left(x_{i}\right)+\epsilon$, where $\epsilon$ represents the noise characterized by its variance $\tau_{i}^{2}$ and $f\left(x_{i}\right)$ the "true" unknown function value. Consequently, the diagonal terms of the covariance matrix are modified to $C_{N}+\Delta$ with $\Delta=\operatorname{diag}\left(\left[\tau_{1}^{2}, \tau_{2}^{2}, \ldots, \tau_{N}^{2}\right]\right)$. Thus, the Gaussian model is updated to:

$$
\hat{f}_{N+1}=K_{N+1}^{\top}\left(C_{N}+\Delta\right)^{-1} F_{N}, \quad \hat{\sigma}_{f_{N+1}}^{2}=\kappa-K_{N+1}^{\top}\left(C_{N}+\Delta\right)^{-1} K_{N+1} .
$$

As will be shown in the following sections, the introduction of noisy observations is necessary to achieve a satisfactory convergence of an optimization procedure based on an error-prone cost function.

\section{Test-case description}

The test-case proposed here corresponds to a classical case of detached flow: we consider the two-dimensional flow over a backwards facing step, in the conditions described in the experiments carried out by Driver \& Seegmiller [7]. Reference computations can be found in [24]. The objective of the actuation is the reduction of the separation length.

The geometry of the computational domain, including the actuator, is represented on figure 1. Computational parameters are set according to the experimental data: $h=0.0127 \mathrm{~m}, U_{\infty}=44.2 \mathrm{~m} / \mathrm{s}$, the boundary layer thickness at the inlet is $\delta_{B L}=0.019 \mathrm{~m}$. The Reynolds number based on this reference length is $R e=5000$. As boundary conditions, we impose at inlet a turbulent velocity profile corresponding to experiments, and a uniform pressure condition at outlet.

Three meshes are generated to assess grid convergence, counting respectively 29778, 45805, 77064 nodes, and 29923, 45953 and 77198 cells. All grids include a refined area between the step and the location $9 h$, as illustrated in figure 2. The distance between the wall and the first node is set to fulfill the condition $y^{+} \leq 1$ at all locations, for all closures. 
The actuator has a width of value $\mathrm{h} / 10$ and is introduced at a location very close to the step corner $(h / 50)$, generating a vertical suction / blowing actuation according to equation (3).

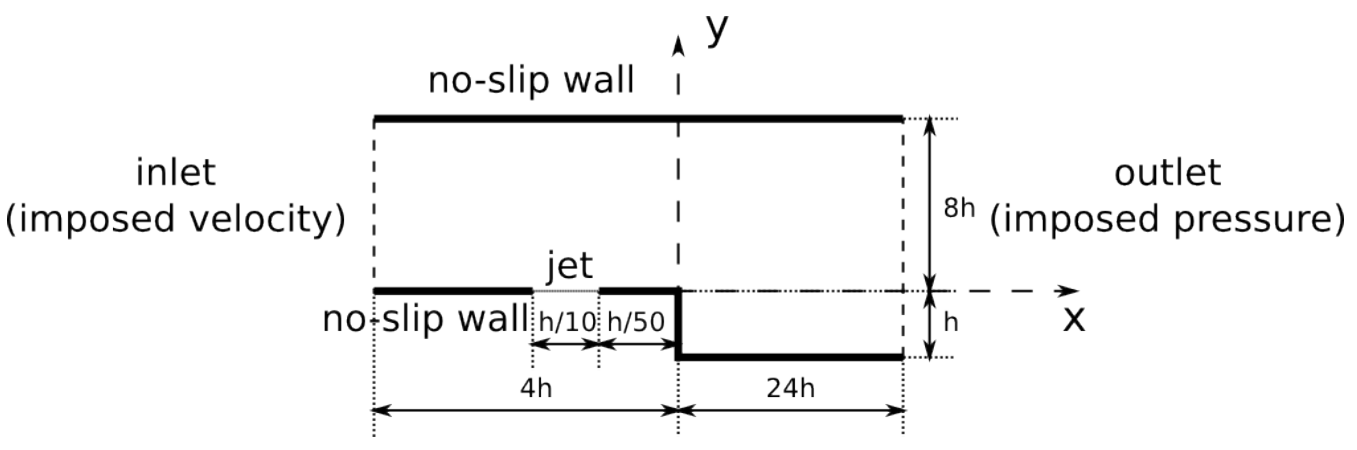

Figure 1: Geometry of the backward facing step case.

\section{Baseline flow}

Firstly, the characteristics of the flow without actuation are compared for the different turbulence closures considered in this study. In this context, the computations converge towards stationary solutions, without KelvinHelmholtz type shear layer instabilities or flapping motions which can be observed experimentally or using LES simulations for instance. A grid convergence study is carried out for all closures, as illustrated in figure 3 , in terms of separation length. As seen, the discretization error is far lower than the modeling error.

Streamlines are depicted on figure 4 and velocity profiles on figure 5, for the finest grid. A comparison of the separation length is provided by table 1 . Although it deals with a simple test-case, without actuation, turbulence closure plays already a critical role in the flow prediction. In particular, EASM closure overestimates the separation length, while Launder-Sharma $k-\epsilon$ one predicts a too small recirculation region, with respect to experiental measurements. Actually, the discrepancy between the different closures was expected. In the next sections, we quantify the effects of the actuation and observe if some common trends can be established, despite of this initial discrepancy. 


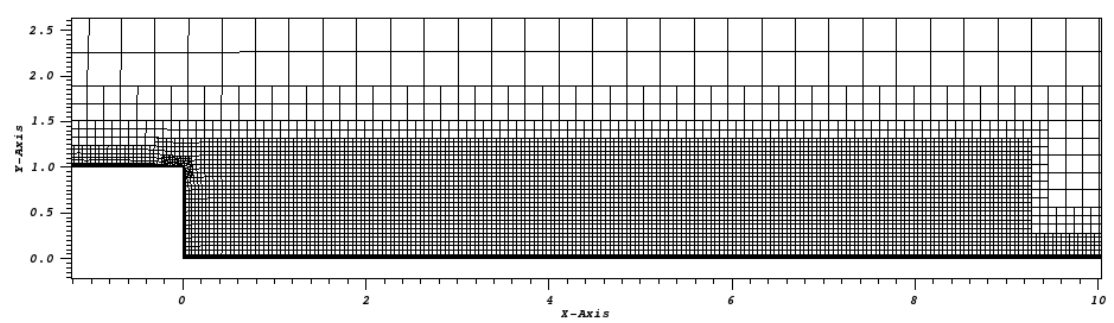

(a) Coarse grid.

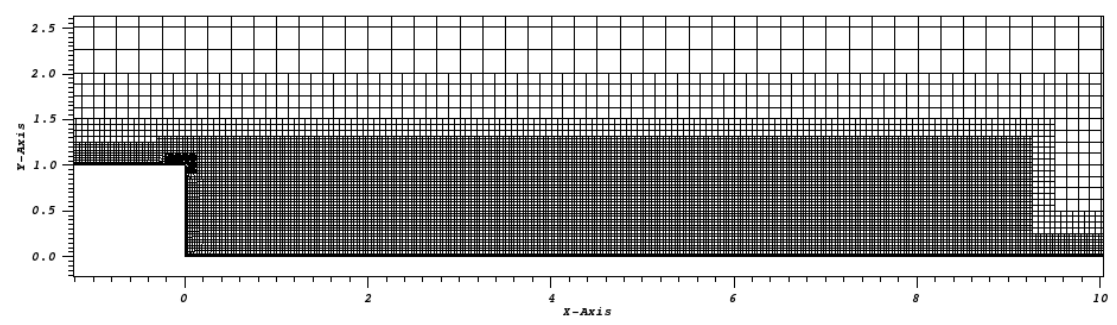

(b) Medium grid.

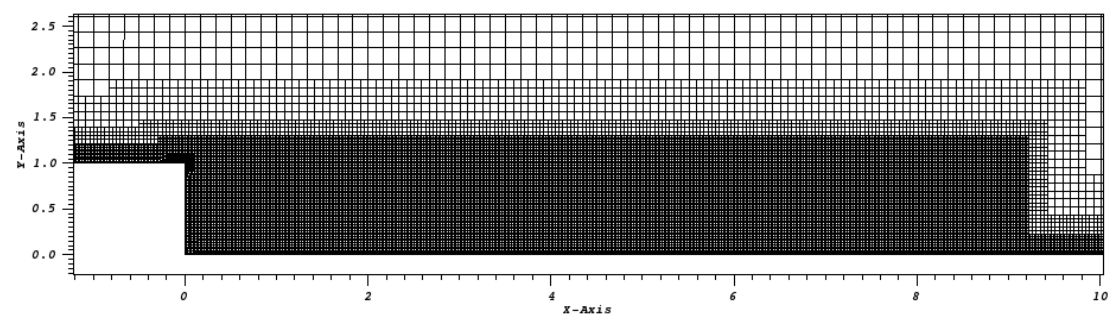

(c) Fine grid.

Figure 2: Grids used for mesh convergence study.

\begin{tabular}{|c|c|c|c|}
\hline Exp. & LS $k-\varepsilon$ & SST $k-\omega$ & EASM \\
\hline 6.26 & 5.41 & 6.37 & 7.56 \\
\hline
\end{tabular}

Table 1: Comparison of separation length $l / h$ without actuation.

\section{Controlled flow validation}

Each simulation of actuated flow is performed starting from the steady state flow found without actuation. Unsteady computations are carried out until transient effects have vanished. The time step employed is scaled on the actuation frequency, so that all configurations count the same number of time 


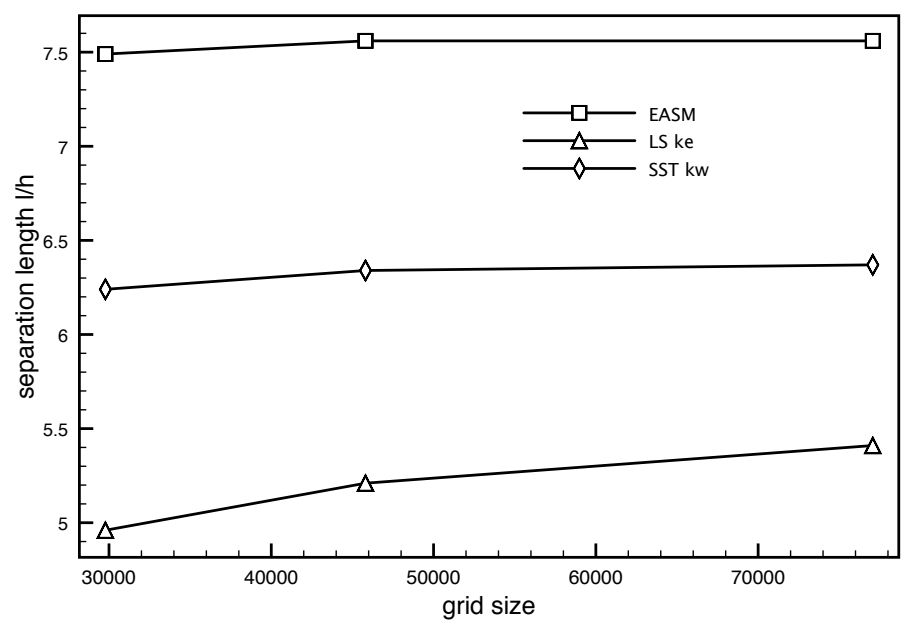

Figure 3: Grid convergence study without actuation.

steps per actuation period. For each time step, a reduction of three orders of non-linear residuals is used as stopping criterion. The separation length is computed afterwards, from the time-averaged flow, on the basis of a fourperiod integration. The separation length is estimated by locating the point where the skin friction vanishes. The parameters of the actuation are the frequency $f_{j}$ and the amplitude $U_{j}$ in equation (3). They are allowed to vary in the following intervals: $4 \mathrm{~m} / \mathrm{s} \leq U_{j} \leq 50 \mathrm{~m} / \mathrm{s}$ and $50 \mathrm{~Hz} \leq f_{j} \leq 1000 \mathrm{~Hz}$.

A convergence study, in terms of grid size and time step, is first performed to assess the computations. Since the optimization criterion is the separation length, this quantity is used for the comparisons. In the perspective of optimization, the grid and the time step should be selected in such a way that the variations of the separation length, due to a change of actuation parameters, are accurately quantified. Therefore, a set of actuation configurations are compared. This study is conducted for the EASM closure only, which is expected to be the most demanding one. Figure 6 presents the evolution of the separation length as the grid size increases, for a fixed fine time step. Figure 7 shows the evolution as the time step is reduced, for the finest grid. Eight actuation configurations are selected randomly in the admissible domain. As can be seen, the medium grid provides satisfactory results: for all configurations except one, the separation length values 


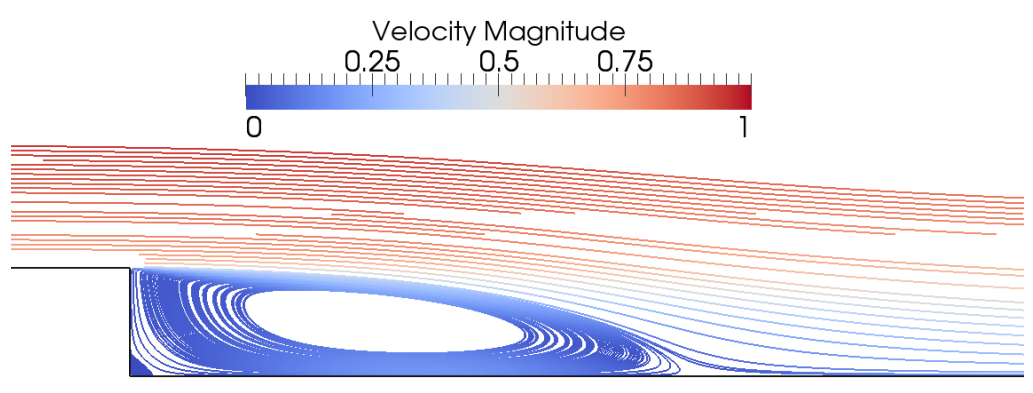

(a) Launder-Sharma $k-\varepsilon$ closure.

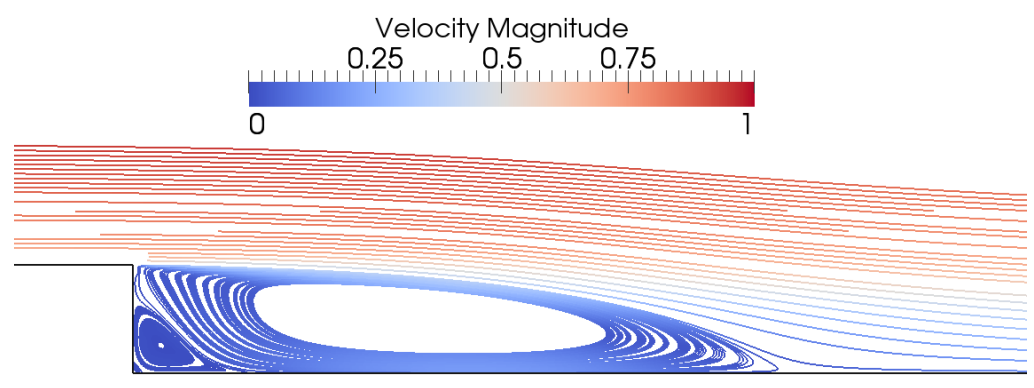

(b) Menter SST $k-\omega$ closure.

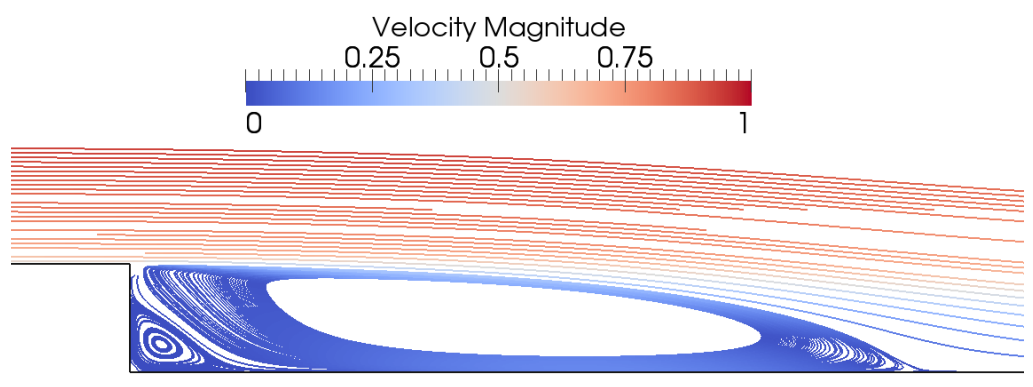

(c) EASM closure.

Figure 4: Comparison of velocity streamlines for different turbulence closures for the flow without actuation.

evolve similarly, as the grid size is refined from the medium mesh to the fine mesh. Even if a full convergence of the value is not achieved, the ranking is correctly predicted. Regarding the time step selection, 64, 128 and 256 time steps per actuation period are tested. As observed, the recirculation length values vary also similarly, as the time step is refined from 128 to 256 


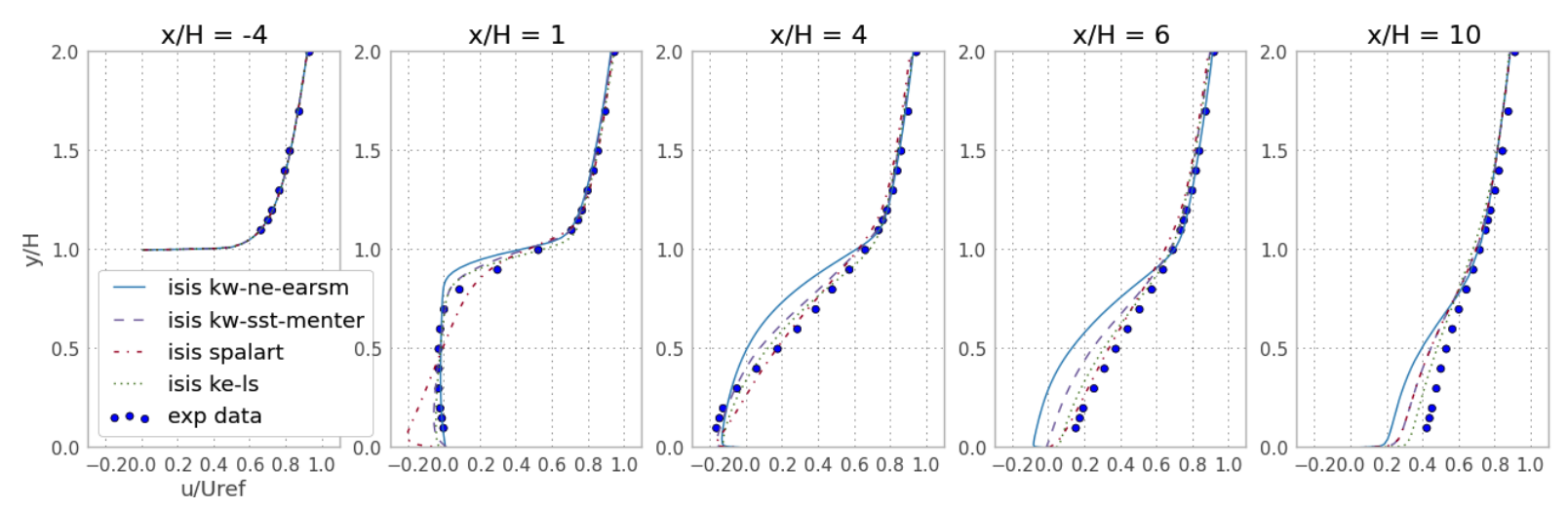

Figure 5: Velocity profiles at $x=-4,1,4,6,10$ for different turbulence closures without actuation.

time steps per period, except for two configurations, generating a switch of two neighboring values. On this basis, the medium grid size and 128 time steps per period are chosen for the optimization exercises. As shown below, this choice is validated a posteriori: the improvement obtained during the optimization, as well as the discrepancy observed between the closures, are far larger than the discretization error reported here.

\section{Optimization of actuation parameters}

The objective of the optimization is the reduction of the separation length, by choosing efficient frequency and amplitude parameters. As described above, the evaluation of the separation length is achieved with great care. However, the estimation of this quantity cannot be more accurate than the local grid size. The resulting noise in the functional evaluation may affect the Gaussian process model [21]. Therefore, we introduce in the model a uniform observation variance of value $\tau^{2}$.

As explained above, the optimization procedure starts from a DOE phase, composed of only six points, that are selected according to a Latin Hypercube Sampling (LHS) [33]. Then, additional points are inserted in the Gaussian process model, according to the lower bound merit function. Three points are considered at each step, corresponding to $\rho=0,1$ and 2. About 12 enrichment steps are performed before convergence of the optimization procedure, corresponding to a total of about 40 simulations per optimization exercise. 
At first, we illustrate the impact of the noisy observation approach: we consider the database collected during the whole optimization procedure, using the $k-\epsilon$ closure, and we construct a Gaussian process model for different values of the observation standard deviation, ranging from $\tau=$ $10^{-4} h$ to $\tau=10^{-1} h$, as shown in figure 8 . If the cost function evaluations are supposed to be exact, or if the observation standard deviation is below a given threshold, the model generated is highly oscillating and stretched. This results from the presence of errors in the database values. On the contrary, for an observation standard deviation above $10^{-2} h$, the model is more regular and does not evolve significantly as the standard deviation is increased. In [21], we established that this threshold value corresponds to the cell size at the reattachment location, which makes sense for a cost function based on the separation length. Note that the noise level is far lower than the separation length and, therefore, does not influence the results presented below, although filtering is a key ingredient to obtain a correct Gaussian Process model and a satisfactory convergence to the optimum.

Figure 9 shows the Gaussian process models obtained using the different turbulence closures, as well as the configurations computed. As can be seen, the initial set of simulations covers the whole admissible domain, while the next evaluations converge towards the optimal parameters. The results obtained with the three closures exhibit some similarities. In particular, a single minimum is identified by the Gaussian process models and the shapes of these models look close to each other. For Launder-Sharma $k-\epsilon$ and EASM closures, the optimum is located in the same region corresponding to medium actuation parameters. However, for the Menter SST $k-\omega$ closure, the minimum corresponds to higher frequency and amplitude. One can underline that, for these three closures, the Gaussian process model exhibits a large flat region for which the actuation is efficient. Nevertheless, the precise values of the optimized control parameters are significantly different, as shown in table 2. Obviously, the "true" optimal actuation value is unknown.

A cross-validation exercise is then performed: the optimal configuration found by using a closure is simulated with other models. Results are shown in table 3. We note that the minimum value for each closure is obtained for the configuration optimized with the same closure. This is consistent, since the latter value is supposed to be the optimal one.

Beyond these observations regarding the best actuation found by the different closures, we propose to compare the characteristics of the corresponding flows, to try to draw some conclusions. In this perspective, the vorticity 


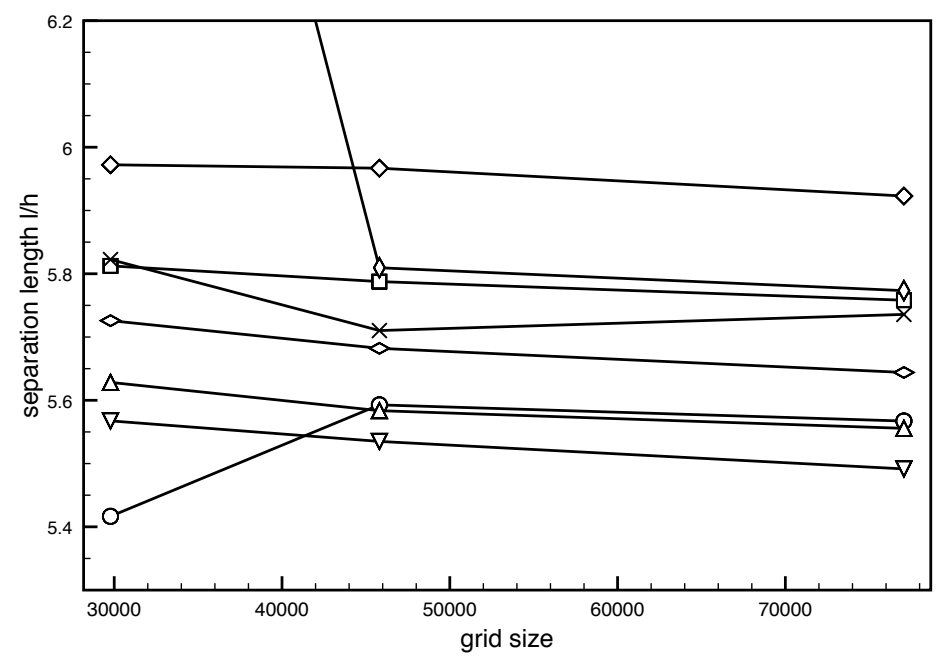

Figure 6: Grid convergence study for eight different actuations (EASM closure). From top to bottom for the finest grid: $(44.8 \mathrm{~m} / \mathrm{s}, 303 \mathrm{~Hz})(50.0 \mathrm{~m} / \mathrm{s}, 810 \mathrm{~Hz})(34.6 \mathrm{~m} / \mathrm{s}$, $1000 \mathrm{~Hz})(7.0 \mathrm{~m} / \mathrm{s}, 493 \mathrm{~Hz})(46.9 \mathrm{~m} / \mathrm{s}, 683 \mathrm{~Hz})(13.2 \mathrm{~m} / \mathrm{s}, 936 \mathrm{~Hz})(22.4 \mathrm{~m} / \mathrm{s}, 873 \mathrm{~Hz})$ $(31.6 \mathrm{~m} / \mathrm{s}, 556 \mathrm{~Hz})$.

\begin{tabular}{|c|c|c|c|}
\hline & $k-\varepsilon$ LS & $k-\omega$ SST & $k-\omega$ EASM \\
\hline Frequency $(\mathrm{Hz})$ & 492 & 828 & 653 \\
Amplitude (m/s) & 31 & 18 & 25 \\
Length / Length ini & 0.72 & 0.84 & 0.70 \\
\hline
\end{tabular}

Table 2: Comparison of optimal parameters and recirculation length found.

\begin{tabular}{|c|c|c|c|}
\hline & $k-\varepsilon$ LS & $k-\omega$ SST & $k-\omega$ EASM \\
\hline Optimized with $k-\varepsilon$ LS & $\mathbf{3 . 9 2}$ & 5.73 & 5.33 \\
\hline Optimized with SST $k-\omega$ & 4.03 & $\mathbf{5 . 3 5}$ & 5.34 \\
\hline Optimized with EASM & 3.95 & 5.41 & $\mathbf{5 . 2 7}$ \\
\hline
\end{tabular}

Table 3: Cross-comparison of recirculation lengths found for optimized configurations and evaluated using different closures.

and turbulent viscosity fields are plotted for the best actuations found in figures 10 to 12 . In each case, four snapshots are proposed, corresponding to the the zero blowing phase $(\Phi=0)$, maximum blowing phase $(\Phi=\Pi / 2)$, zero suction phase $(\Phi=\Pi)$ and maximum suction phase $(\Phi=3 \Pi / 2)$. For 


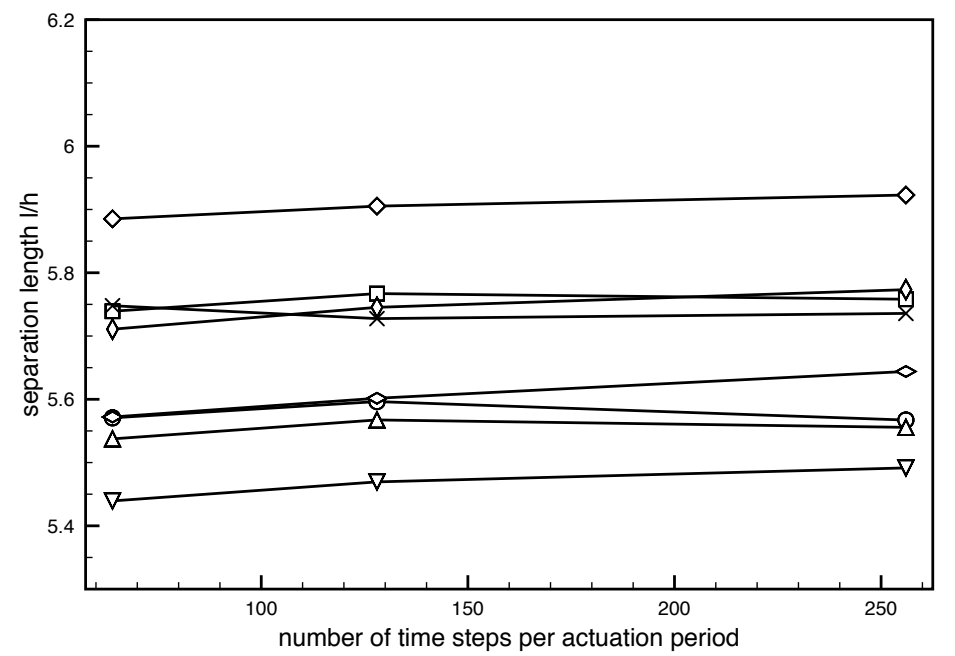

Figure 7: Time step convergence study for eight different actuations (EASM closure). From top to bottom for the smallest time-step: $(44.8 \mathrm{~m} / \mathrm{s}, 303 \mathrm{~Hz})(50.0 \mathrm{~m} / \mathrm{s}, 810 \mathrm{~Hz})$ $(34.6 \mathrm{~m} / \mathrm{s}, 1000 \mathrm{~Hz})(7.0 \mathrm{~m} / \mathrm{s}, 493 \mathrm{~Hz})(46.9 \mathrm{~m} / \mathrm{s}, 683 \mathrm{~Hz})(13.2 \mathrm{~m} / \mathrm{s}, 936 \mathrm{~Hz})(22.4 \mathrm{~m} / \mathrm{s}$ , $873 \mathrm{~Hz})(31.6 \mathrm{~m} / \mathrm{s}, 556 \mathrm{~Hz})$.

the three closures considered here, the best actuation found yields the birth of vortices at the step corner. Note that the resulting flows look similar to Kelvin-Helmholtz instabilities in mixing layers. The three corresponding flows exhibit similar patterns, although the excitation frequency is different for each case. Especially, the most efficient flows predicted by the SST $k-\omega$ and the EASM closures are close to each other. Nevertheless, the nonlinearities of the EASM closure generates a more vortical flow, with more intense variations of the turbulent viscosity. The best configuration found by using the Launder-Sharma $k-\varepsilon$ closure has a lower actuation frequency. The corresponding flow exhibits vortices with larger span and a far higher turbulent viscosity level.

Finally, we represent in figure 13 the distribution of $\overline{u^{\prime} v^{\prime}}$ at different $\mathrm{x}$ sections, for the three models, for the baseline and optimized configurations. For URANS computations, these profiles include both modeled and resolved parts. Experimental results are also provided for the baseline case [7]. The analyse confirms that a similar control mechanism is exploited by the best actuations found with the SST $k-\omega$, the Launder-Sharma $k-\varepsilon$ and the EASM closures. One can especially notice the generation of turbulent shear stress in 


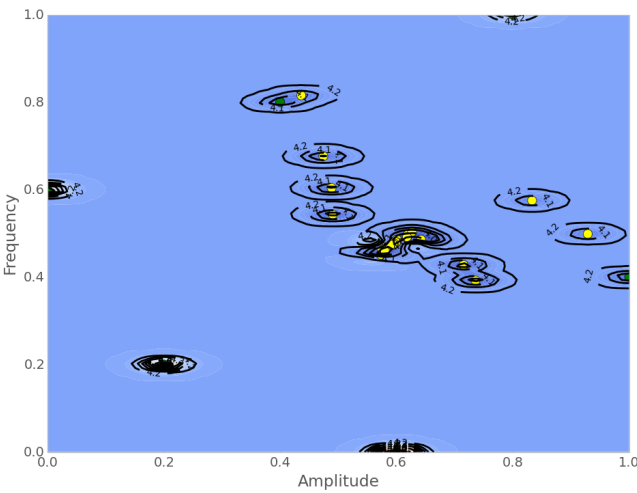

(a) $\tau=10^{-4} h$

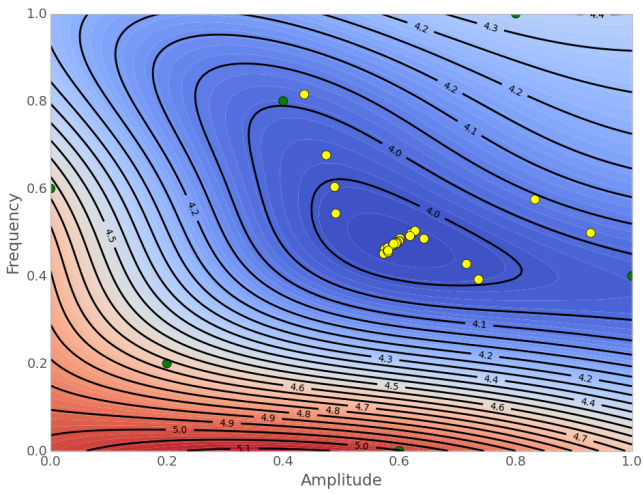

(c) $\tau=10^{-2} h$

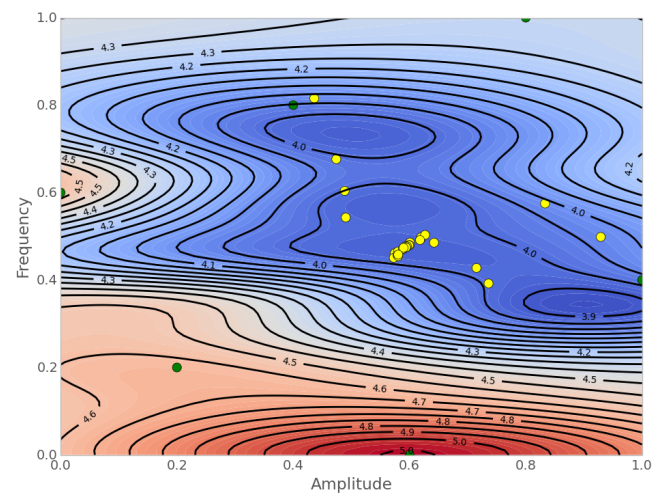

(b) $\tau=10^{-3} h$

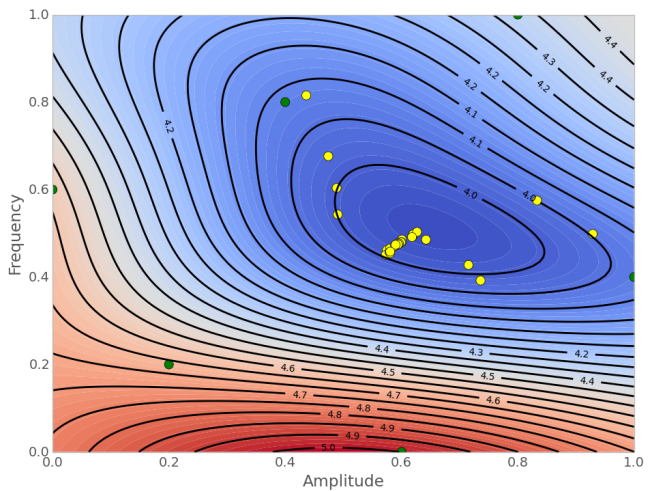

(d) $\tau=10^{-1} h$

Figure 8: Influence of the observation variance $\tau^{2}$ on the Gaussian process model (normalized variables).

the vicinity of the step (section $x=h$ ) and the bottom wall. At the first and second sections the increase is especially noticeable for EASM closure. At downstream section, the profiles are similar to the ones without actuation, but with a slightly higher stress level.

\section{Discussion}

For the test-case studied here, the characteristics of the baseline flow depend significantly on the turbulence closure. This is usual when separated flows are considered. The quantity of interest (separation length), which is also the optimization criterion for active control, exhibits variations of $25 \%$ 
when different closures are tested. This is obviously a difficult context for optimization. However, we underline that optimization could be achieved successfully, despite of these discrepancies, if the different closures provide similar flow changes, as the control parameters are modified. If the same error occurs during the whole optimization procedure, the different closures can yield the same optimum parameters, although the flow predictions differ. This has been already observed in shape optimization exercises related to pressure drop for instance: the optimum shape can be found even if the pressure field is not accurately predicted[2].

Unfortunately, the results presented above show that separated flows with actuation are not a so friendly context. The discrepancies observed for baseline flow yield different search directions and, finally, significantly different optimum parameters. One can conclude that RANS models cannot be employed blindly in a design optimization procedure to determine actuation parameters. Obviously, the "true" optimum parameters are unknown. For the baseline flow, the SST $k-\omega$ closure provides the best separation length prediction, according to the experiments. Nevertheless, it is even not clear if the best parameters predicted by this closure are close to the "true" optimum. The use of LES could provide some reference results, but optimization based on LES is still unaffordable, for computational time reasons.

However, some similarities between the flows with optimal actuation have been reported. This seems to indicate that the different optimization exercises yield the same flow characteristics, whatever the closure. If this is confirmed, RANS models can still be used in a first design step, to determine the characteristics of efficient flow actuations.

\section{Conclusion}

The extension of active control methodologies to industrial cases will require optimizing actuator parameters. Presently, RANS models are the only admissible methods to achieve such objectives. In this perspective, we have coupled a RANS solver with an optimization algorithm based on a statistical learning and applied the resulting tool to the optimization of the actuation frequency and amplitude, for a backward facing step. The cost functional to minimize is the time-averaged separation length. 
Baseline and actuated flows have been carefully validated using a grid and time step refinement study. Then, some optimization exercises have been carried out, by using different turbulence closures. It has been found that the different closures yield significantly different optimum parameters. However, some similarities between the flows with optimum actuation have been reported, indicating that RANS models could still be employed to determine the characteristics of efficient flow actuation.

To overcome the limitation of RANS models, the development of optimization strategies based on hybrid evaluations, mixing RANS and more sophisticated closures like DES, seems to be a necessity in the perspective of solving more complex active control problems.

\section{Acknowledgements}

This study is partially supported by the 7th Framework Program of the European Union, project number 266326 "MARS". 


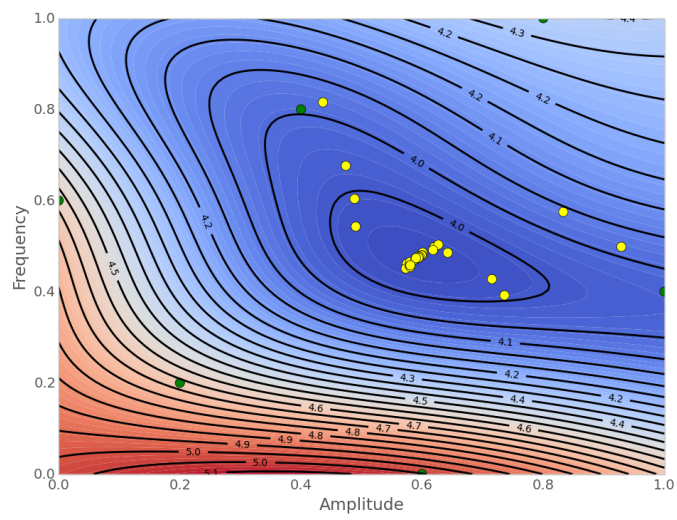

(a) Launder-Sharma $k-\epsilon$ closure.

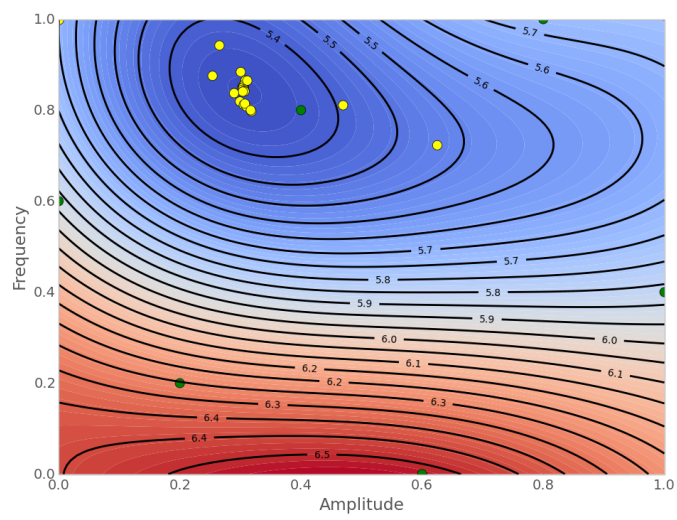

(b) Menter SST $k-\omega$ closure.

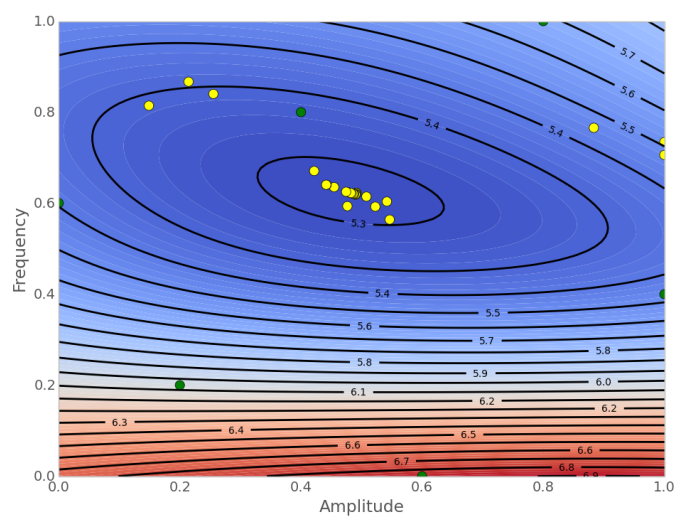

(c) EASM closure.

Figure 9: Gaussian process models of the separation length w.r.t. actuation frequency and amplitude (normalized variables), and simulated points. Green points correspond to initial Latin Hypercube Sampling. 


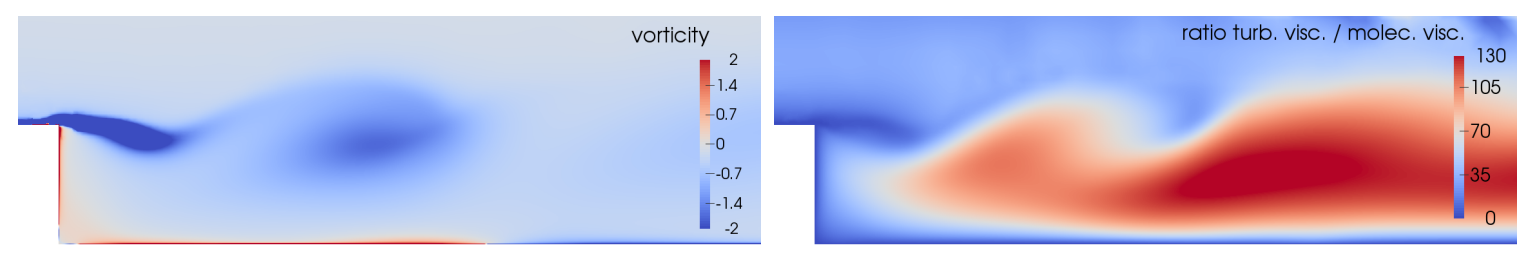

(a) $\Phi=0$.

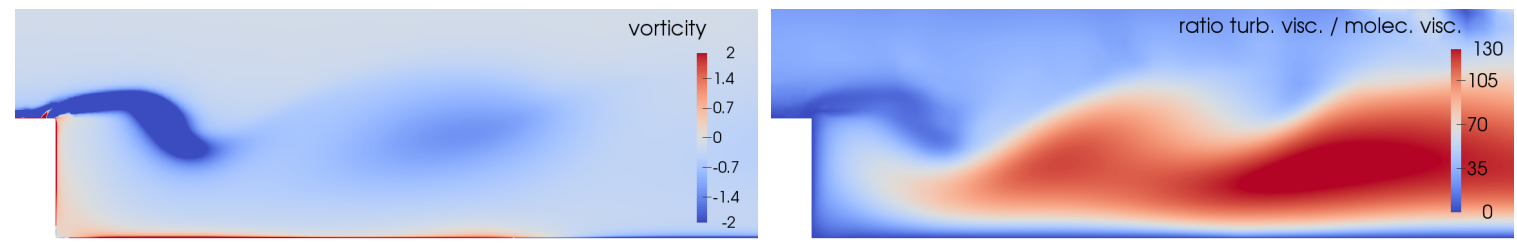

(b) $\Phi=\Pi / 2$.

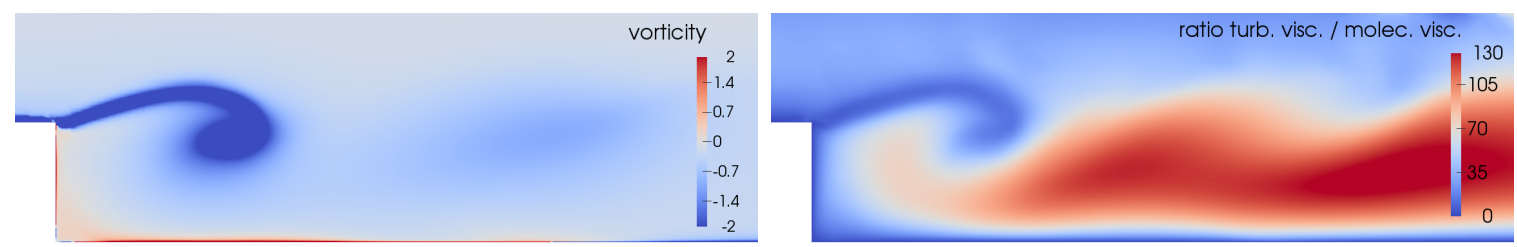

(c) $\Phi=\Pi$.

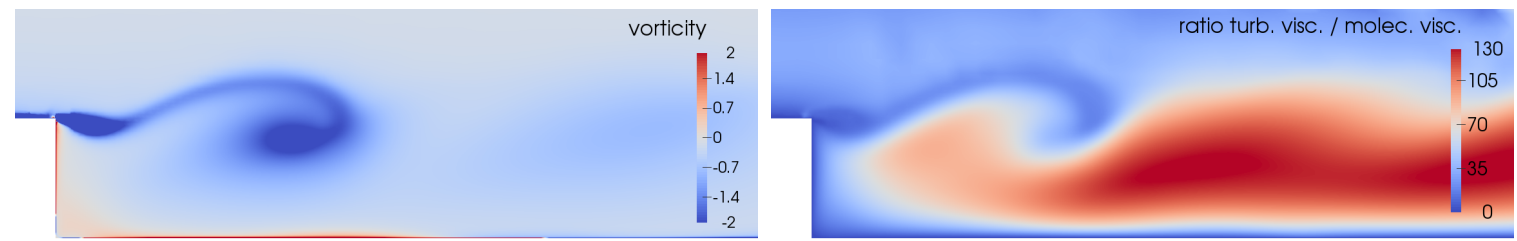

(d) $\Phi=3 \Pi / 2$.

Figure 10: Vorticity (left) and turbulent viscosity (right) fields for different actuation phases for control parameters optimized with Launder-Sharma $k-\epsilon$ closure $(492 \mathrm{~Hz}$, $31 \mathrm{~m} / \mathrm{s})$. 

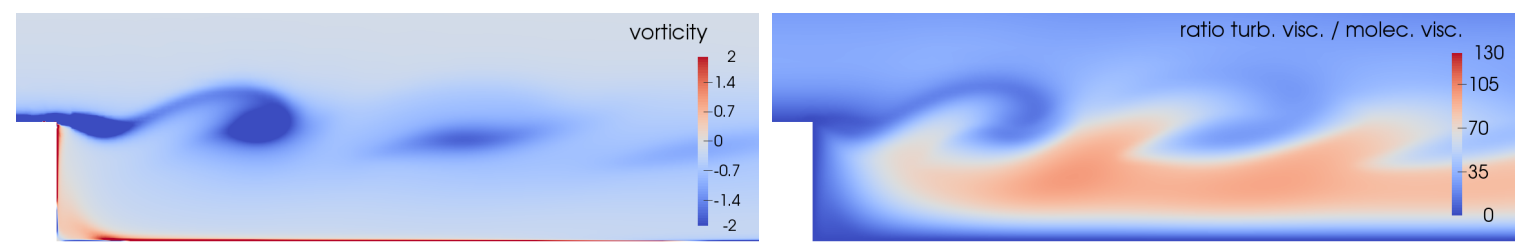

(a) $\Phi=0$.

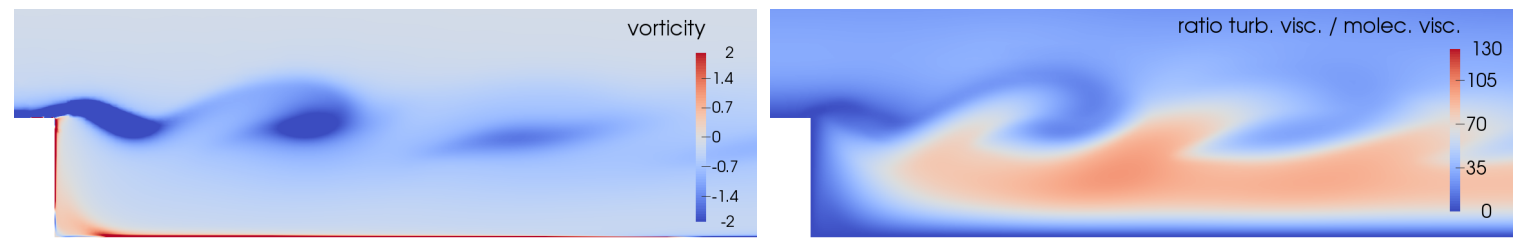

(b) $\Phi=\Pi / 2$.

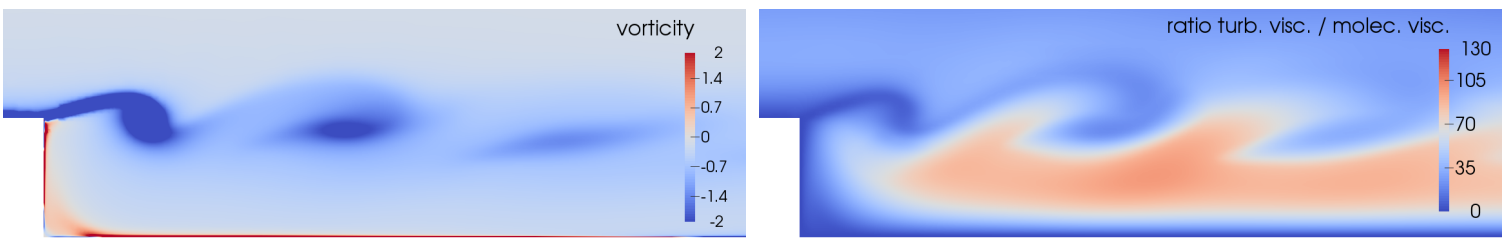

(c) $\Phi=\Pi$.

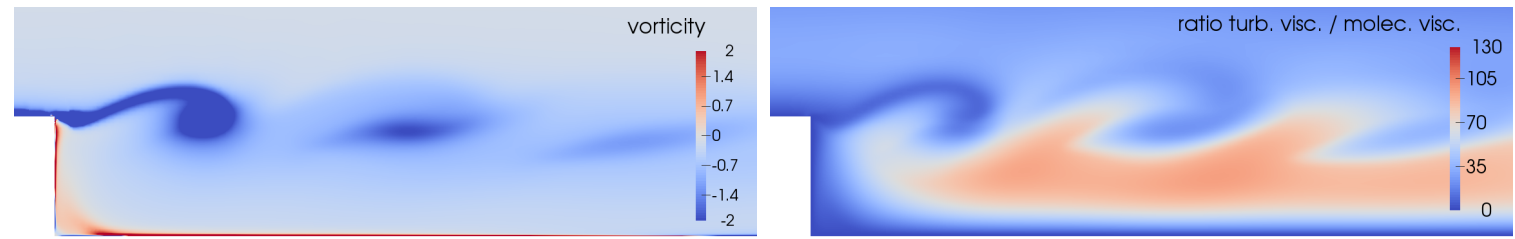

(d) $\Phi=3 \Pi / 2$.

Figure 11: Vorticity (left) and turbulent viscosity (right) fields for different actuation phases for control parameters optimized with Menter SST $k-\omega$ closure $(828 \mathrm{~Hz}, 18 \mathrm{~m} / \mathrm{s})$. 

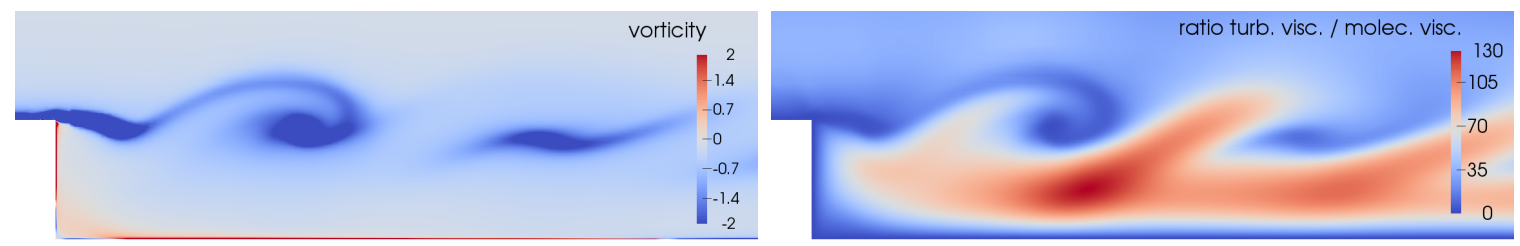

(a) $\Phi=0$.

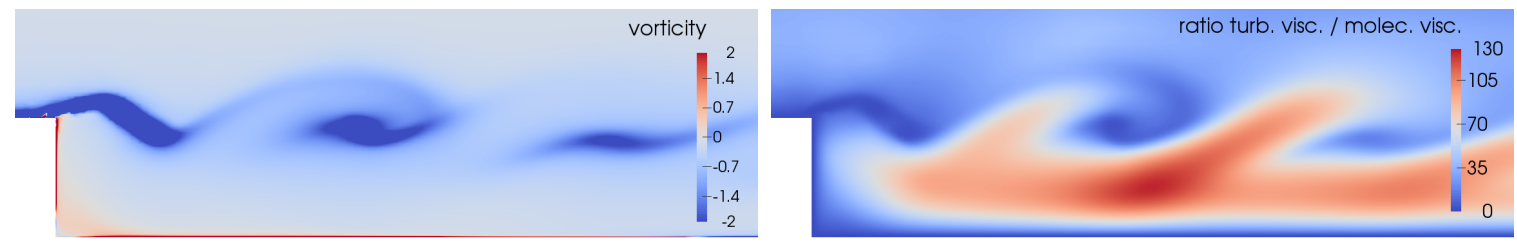

(b) $\Phi=\Pi / 2$.

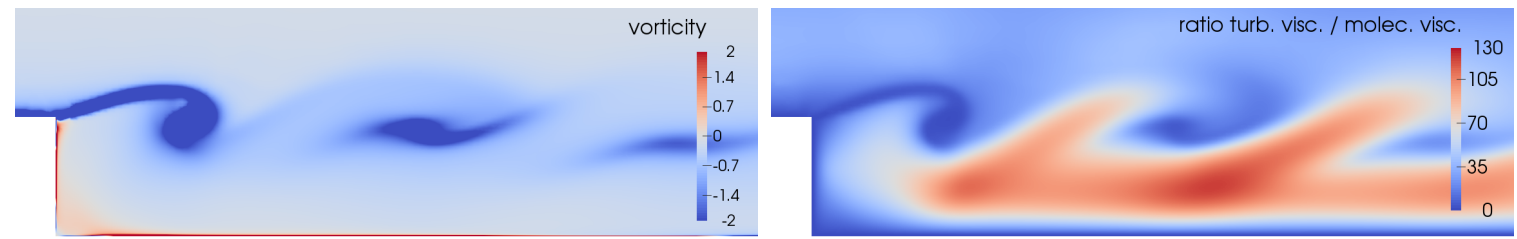

(c) $\Phi=\Pi$.

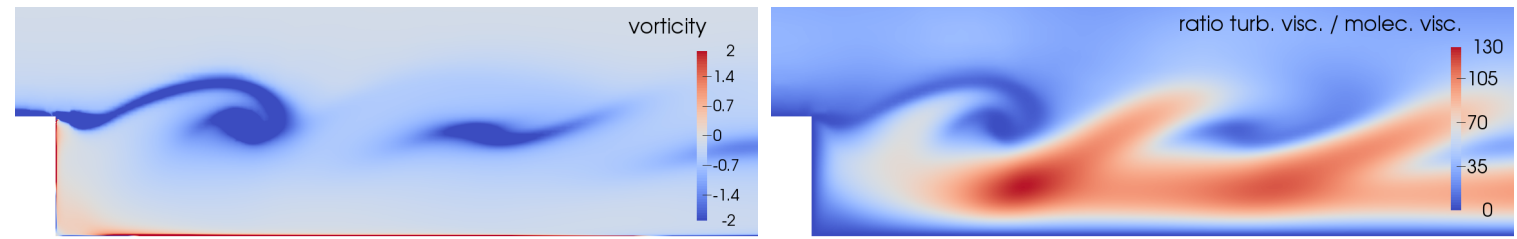

(d) $\Phi=3 \Pi / 2$.

Figure 12: Vorticity (left) and turbulent viscosity (right) fields for different actuation phases for control parameters optimized with EASM closure $(653 \mathrm{~Hz}, 25 \mathrm{~m} / \mathrm{s})$. 

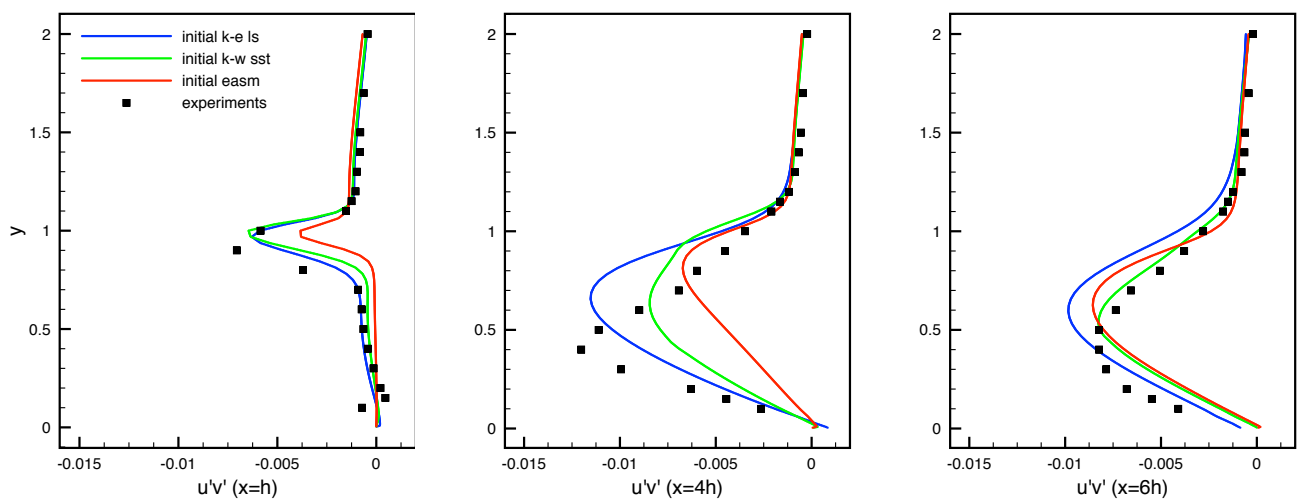

(a) Baseline configuration without actuation.
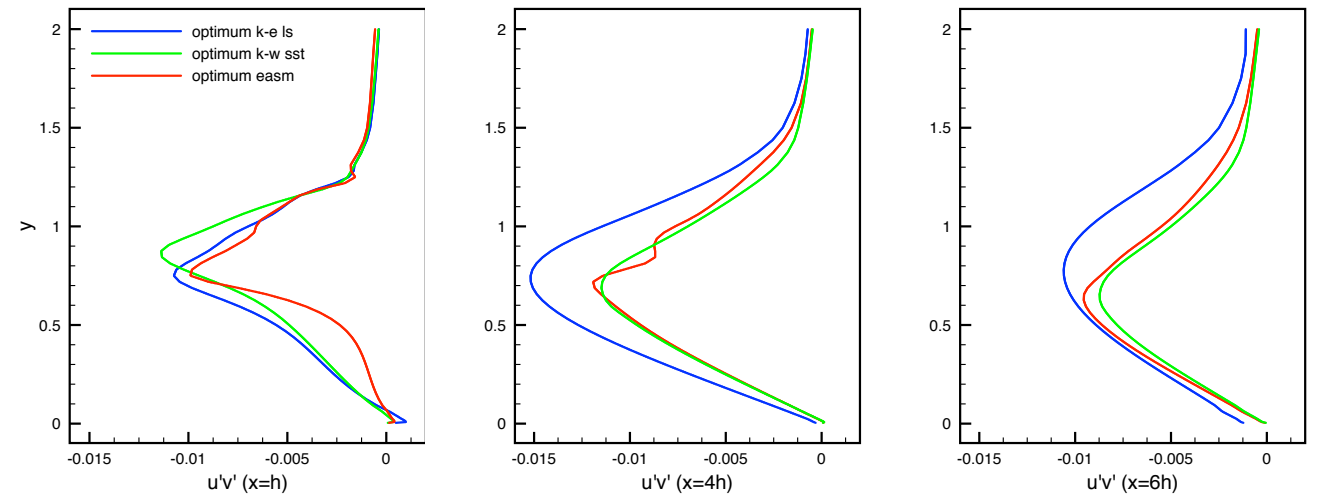

(b) Best actuation for each closure.

Figure 13: Distribution of $\overline{u^{\prime} v^{\prime}}$ at different $\mathrm{x}$-sections, for different closures. 
[1] D. Büche, N. N. Schraudolph, and P. Koumoutsakos. Accelerating evolutionary algorithms with gaussian process fitness function models. IEEE Tran. on Systems, Man, and Cybernetics - Part C: Applications and Reviews, 35(2):183-194, 2005.

[2] J. Burman and B.R. Gebart. Influence from numerical noise in the objective function for flow design optimization. Int. J. of Numerical Methods for Heat and Fluid Flow, 11(1):6-19, 2001.

[3] A. Carnarius, F. Thiele, E. Oezkaya, A. Nemili, and N. Gauger. Optimization of active flow control of a naca 0012 airfoil by using a continuous adjoint approach. In European Congress on Computational Methods in Applied Sciences and Engineering, Vienna, Austria, 2012.

[4] P. Chandrashekar and R. Duvigneau. Study of some strategies for global optimization using gaussian process models with application to aerodynamic design. Technical Report RR-6964, INRIA, June 2009.

[5] G.B. Deng and M. Visonneau. Comparison of explicit algebraic stress models and second-order turbulence closures for steady flows around ships. In Proc. 7th Int. Conf. on Numerical Ship Hydrodynamics, Nantes, France, 1999.

[6] J.F. Donovan, L.D. Kral, and A.W. Cary. Active flow control applied to an airfoil. AIAA Paper 98-0210, January 1998.

[7] D.M. Driver and H.L. Seegmiller. Features of a reattaching turbulent shear layer in divergent channel flow. AIAA journal, 23(2):163-171, 1985 .

[8] R. Duvigneau and P. Chandrashekar. Kriging-based optimization applied to flow control. Int. J. for Numerical Methods in Fluids, 69(11):1701-1714, 2012.

[9] R. Duvigneau, A. Hay, and M. Visonneau. Optimal location of a synthetic jet on an airfoil for stall control. Journal of Fluid Engineering, 129(7):825-833, July 2007.

[10] R. Duvigneau and M. Visonneau. Optimization of a synthetic jet actuator for aerodynamic stall control. Computers and Fluids, 35:624-638, July 2006. 
[11] R. Duvigneau, M. Visonneau, and G.B. Deng. On the role played by turbulence closures for hull shape optimization at model and full scale. Journal of Marine Science and Technology, 8(1):11-25, June 2003.

[12] J.A. Ekaterinaris. Active flow control of wing separated flow. ASME FEDSM'03 Joint Fluids Engineering Conference, Honolulu, Hawai, USA, July 6-10, 2003.

[13] M. Gad El-Hack, A. Pollard, and J.-P. Bonnet. Flow control: fundamentals and practices. Springer-Verlag, 1998.

[14] E. Garnier, P.Y. Pamart, J. Dandois, and P. Sagaut. Evaluation of the unsteady rans capabilities for separated flow control. Computers $\&$ Fluids, 61:39-45, 2012.

[15] T.B. Gatski and C.G. Speziale. On explicit algebraic stress models for complex turbulent flows. Journal of Fluid Mechanics, 254(1):59-78, 1993.

[16] J.L. Gilarranz, L.W. Traub, and O.K. Rediniotis. Characterization of a compact, high power synthetic jet actuator for flow separation control. AIAA Paper 2002-0127, September 2002.

[17] J. W. He, R. Glowinski, R. Metcalfe, A. Nordlander, and J. Periaux. Active control and drag optimization for flow past a circular cylinder: I. oscillatory cylinder rotation. Journal of Computational Physics, 163(1):83-117, 2000.

[18] A. Jameson. Time dependent calculations using multigrid, with application to unsteady flows past airfoils and wings. In 10th Computational Fluid Dynamics Conference, Honolulu, Hawai., 1991.

[19] D.R. Jones. A taxonomy of global optimization methods based on response surfaces. Journal of Global Optimization, 21:345-383, 2001.

[20] L.D. Kral, J.F. Donovan, A.B. Cain, and A.W. Cary. Numerical simulation of synthetic jet actuators. In AIAA Paper 97-1824, 1997.

[21] J. Labroquère, R. Duvigneau, and E. Guilmineau. Impact of turbulence closures and numerical errors for the optimization of flow control devices. In 21th AIAA Computational Fluid Dynamics Conference, San Diego, USA, 2013. 
[22] B.E. Launder and D.B. Spalding. The numerical computation of turbulent flows. Computer Methods in Applied Mechanics and Engineering, 3(2):269-289, 1974.

[23] J. Laurenceau and P. Sagaut. Efficient response surfaces of aerodynamic functions with kriging and cokriging. AIAA Journal, 46(2):498-507, 2008.

[24] H. Le, P. Moin, and J. Kim. Direct numerical simulation of turbulent flow over a backward-facing step. Journal of Fluid Mechanics, 330(1):349-374, 1997.

[25] F.R. Menter. Two-equation eddy-viscosity turbulence models for engineering applications. AIAA Journal, 32(8):1598-1605, 1994.

[26] Victor Picheny, David Ginsbourger, and Yves Richet. Noisy expected improvement and on-line computation time allocation for the optimization of simulators with tunable fidelity. In 2nd Int. Conf. on Engineering Optimization, Lisbon, Portugal, 2010.

[27] V. Przulj and B. Basara. Bounded convection schemes for unstructured grids. AIAA Paper 2001-2593, 2001.

[28] P. Queutey and M. Visonneau. An interface capturing method for freesurface hydrodynamic flows. Computers \& fluids, 36(9):1481-1510, 2007.

[29] C.L. Rhie and W.L. Chow. A numerical study of the turbulent flow past an isolated airfoil with trailing edge separation. In $A I A A$ Paper 82-0998, AIAA/ASME 3rd joint Thermophysics, Fluids, Plasma and Heat Transfer Conference, 1982.

[30] B.D. Ritchie, D.R. Mujumdar, and J.M. Seitzman. Mixing in coaxial jets using synthetic jet actuators. In AIAA Paper 2000-0404, 2000.

[31] D.P. Rizetta, M.R. Visbal, and M.J. Stanek. Numerical investigation of synthetic jet flowfields. In AIAA paper 98-2910, 1998.

[32] C. L. Rumsey, T. B. Gatski, W. L. Sellers, V. N. Vatsa, and S. A. Viken. Summary of the 2004 Computational Fluid Dynamics Validation Workshop on synthetic jets. AIAA Journal, 44(2):194-207, 2006. 
[33] J. Sacks, W.J. Welch, T.J. Mitchell, and H.P. Wynn. Design and analysis of computer experiments. Statistical Science, 4(4):409-435, 1989.

[34] A. Seifert, A. Darabi, and I. Wygnanski. Delay of airfoil stall by periodic excitation. AIAA Journal, 33(4):691-707, July 1996.

[35] R.N. Sharma. Fluid-dynamics-based analytical model for synthetic jet actuation. AIAA Journal, 45(8):1841-1847, 2007.

[36] B. Smith and A. Glezer. Vectoring and small-scale motions effected in free shear flows using synthetic jet actuators. In AIAA Paper 97-0213, 1997.

[37] V. N. Vatsa and E. Turkel. Simulation of synthetic jets using unsteady Reynolds averaged Navier-Stokes equations. AIAA Journal, 44(2):217$224,2006$.

[38] A. Zymaris, D. Papadimitriou, K. Giannakoglou, and C. Othmer. Optimal location fo suction or blowing jets using the continuous adjoint approach. In European Congress on Computational Methods in Applied Sciences and Engineering ECCOMAS 2010 Lisbon, 2010. 